\section{G199(P) COMPARING THE ACCCURACY OF DELIVERY OF A SUSTAINED INFLATION TO INFLATION BREATHS ON A NEONATAL MANNEQUIN}

${ }^{1} \mathrm{~K}$ Hunt, ${ }^{2} \mathrm{R}$ Ling, ${ }^{2} \mathrm{~K}$ Ali, ${ }^{2} \mathrm{~T}$ Dassios, ${ }^{1} \mathrm{~A}$ Milner, ${ }^{1} \mathrm{~A}$ Greenough. ${ }^{1}$ Women and Children's Health, School of Life Course Sciences, King's College London, London, UK; ${ }^{2}$ Neonatal Intensive Care Centre, King's College Hospital NHS Foundation Trust, London, UK

\subsection{6/archdischild-2018-rcpch.194}

Aims The Neonatal Life Support (NLS) guidelines currently recommend delivery of five inflation breaths (IB) each lasting two to three seconds. ${ }^{1}$ In practise, however, doctors often fail to deliver the recommended duration. ${ }^{2}$ There has been increasing interest in delivery of a sustained inflation $(\mathrm{SI}=15 \mathrm{~s})$ as initial resuscitation of prematurely-born infants. Our aim was to investigate how accurately neonatal doctors could deliver an SI compared to IB using a resuscitation mannequin.

Methods Doctors were invited to deliver five IB (each five seconds in duration) and a fifteen second SI to a neonatal mannequin. A respiratory function monitor was used to assess the duration of the inflations. Recordings were made after the doctors had the opportunity to practise using the equipment and delivering an SI. All were trained in NLS.

Results Twenty four doctors took part in the study.

\begin{tabular}{ll} 
Abstract G199(P) Table 1 & \\
\hline Median (range) duration of IB (seconds) & Median (range) duration of SI (seconds) \\
\hline $2.2(1.1-3.8)$ & $15.3(11.5-22.0)$ \\
\hline
\end{tabular}

The median error for IB was $-0.8 \mathrm{~s}$, that is, on average, each inflation was $0.8 \mathrm{~s}$ too short and for SI the median error was $+0.34 \mathrm{~s}$, that is, on average, each inflation was $0.34 \mathrm{~s}$ too long. The magnitude of error was significantly higher for IB than SI (26\% versus $5.7 \%$ respectively, $\mathrm{p}=0.001)$.

To further compare the variability in the two techniques the IB results were divided by three and the SI results by 15 . The interquartile range for IB was $0.59 \mathrm{~s}$ and for SI was $0.25 \mathrm{~s}$, demonstrating much closer clustering of results around the median for the SIs (Table 1).

Conclusions A fifteen second SI was delivered more accurately than three second inflation breaths by neonatal doctors using a mannequin. Studies of neonatal resuscitation should examine the accuracy with which the techniques are applied.

\section{REFERENCES}

1. (UK) RC. NLS Guidelines (Resuscitation Council). resus.org.uk

2. Murthy V, et al. Eur J Pediatr 2012;171:843-6.

\section{G200(P) PARENTAL VIEWS ON ATTENDING WARD ROUNDS IN A NEONATAL INTENSIVE CARE UNIT}

${ }^{1} \mathrm{C}$ Caldwell, ${ }^{2} \mathrm{~V}$ MacBean, ${ }^{2} \mathrm{~A}$ Greenough. ${ }^{1}$ Neonatal Intensive Care Centre, King's College Hospital NHS Foundation Trust, London, UKi ${ }^{2}$ School of Life Course Sciences, Faculty of Life Sciences and Medicine, King's College London, London, UK

\subsection{6/archdischild-2018-rcpch. 195}

Aim The National Neonatal Audit Project will collect data on whether a parent attended a ward round during their baby's admission to a neonatal unit. The aim of the current study was to explore whether parents wished to attend ward rounds and if they did attend had they found it useful.

Method One interviewer approached parents of babies who were current inpatients in our NICU during June-August 2017. Questions included basic demographic data, the time and cost incurred in travelling to the unit, what information parents received about the ward rounds, had they attended ward rounds and what was their experience. In addition, they were asked if they wanted more or less access to ward rounds, were there issues of confidentiality and what other sources of information they had accessed. There was also an opportunity for parents to offer any other comments.

Results Twenty of 21 parents approached agreed to be interviewed. The median (range) age of their babies was 14 (3123) days, and median (range) length of stay was 10 (3-123) days. Seventeen were able to identify a nursing handover or doctors' ward round. Fifteen had attended at least one ward round; the mean score was 4.5 out of 5 in terms of usefulness. Thirteen of fifteen described the experience positively. Eighteen said they would like to attend at least one ward round per day, but one commented that the nursing updates were sufficient. Regarding confidentiality, seventeen were 'not bothered' if other people overheard information about their baby, however some mentioned that if the news was bad, they would not want other people to overhear it. Other sources of information were the internet $(n=14)$ and talking to other parents on the unit $(n=18)$, often in the expressing room.

Conclusion Parents recognise the importance of the ward round in making a treatment plan for the day and are keen to hear this at the time it is made. A minority, however, preferred to have an update from the nursing staff.

\section{G201(P) EXPLORING NEW WAYS OF WORKING IN THE NEONATAL UNIT}

T Mitra, L Bramwells. London School of Paediatrics, Health Education England, London, UK

\subsection{6/archdischild-2018-rcpch. 196}

Aim This project has been commissioned by the London School of Paediatrics/Health Education England to explore new ways of working within neonatal units across London with an aim to provide collaborative recommendations on ways to reduce the dependence of service delivery on the paediatric medical workforce by providing a more stable, mixed, neonatal workforce. Workforce issues are not limited to medical rotas; review of national and local data has found that there is a considerable vacancy across the different professional groups nationally and within London. There is a paucity of qualified in speciality (QIS) nurses and a wide variability in the availability of enhanced and advanced roles in neonatal nursing.

Method Common workforce and service delivery issues already acknowledged by professional bodies were identified during site visits and semi-structured interviews with over half of the London neonatal teams.

Results Units spoke of developing different roles which may support the workforce and these have been explored further. It was clear to see a variance in how units have developed operationally to deliver care. Factors have been identified which have a direct impact on the workforce such as: medical and nursing vacancies, culture, transitional care, admission 
prevention, support for junior nursing staff and the development of the non-registered workforce.

Increasing the support for lower dependency care can potentially relieve pressures upstream. Solutions for workforce transformation cannot be considered in isolation. It has become apparent that organisation and service delivery of care has to be considered in tandem for workforce transformation.

Results To further explore:

- Nursing Associates

- Role definition for Enhanced Neonatal Nurse Practitioners

- Development of Non-Medical Supporting Roles

- Development of Associate Specialist/MTIp roles

- Awareness of impact of culture and morale on the workforce

- Investment in clinical supervision, education and training

- Development of networked preceptorship nursing programmes

- Development of Transitional Care

- Implementation of ATAIN programme

- Implementation of Integrated Family Development Care

However, despite all best intentions, these roles and service developments may not have a sustainable effect on vacancies in middle grade doctors or nurses achieving QIS. It is important to recognise this as a limitation.

\section{G202(P) OUTCOMES OF EXTREMELY LOW BIRTH WEIGHT INFANTS - THE EFFECT OF BEING SMALL FOR GESTATIONAL AGE}

${ }^{1} \mathrm{E}$ Charles, ${ }^{1} \mathrm{C}$ Harris, ${ }^{2} \mathrm{~A}$ Hickey, ${ }^{1} \mathrm{~A}$ Greenough. ${ }^{1}$ Women and Children's Health, School of Life Course Sciences, Faculty of Life Sciences and Medicine, King's College London, London, UK; ${ }^{2}$ Neonatal Intensive Care Centre, King's College Hospital NHS Foundation Trust, London, UK

\subsection{6/archdischild-2018-rcpch.197}

Aims Prematurely-born infants who are small for gestational age (SGA) have worse outcomes than those who are born appropriate for gestational age (AGA). ${ }^{1,2}$ There has, however, been little focus on SGA infants born at extremely low birth weights (BW <750 g).

Methods A retrospective study was undertaken of all babies BW <750 gm born between 2012 and 2016. Centiles and zscores were calculated using the UK WHO preterm reference ranges (British 1990 reference data, reanalysed 2009). The infants were divided into SGA $(<10$ th centile) and AGA (10th -90 th centile) groups. Multiple logistic regression analysis was performed to adjust for gestational age to relate individual outcome variables to SGA.

Results Eighty-four infants were included, 35 (42\%) were SGA. The SGA infants were more mature (median gestational age 26.9 versus 24.3 weeks, $\mathrm{p}<0.001$ ), had a lower birth weight centile (median 1 versus 26, p<0.001); their mothers were more likely to have received antenatal steroids $(94 \%$ versus 78\%, $p=0.022)$, had hypertension $(49 \%$ versus $8 \%$, $\mathrm{p}<0.001)$ and be delivered by caesarean section $(69 \%$ versus $8 \%, \mathrm{p}<0.001)$. Neither the mortality $(31 \%$ versus $32 \%)$ nor the incidence of NEC (17\% versus 16\%) differed significantly between the two groups, but more of the SGA developed severe BPD $(p=0.025)$. The SGA infants achieved full enteral feeds at an older postnatal age (median 54 versus 48 days, $\mathrm{p}=0.019)$. The length of stay was similar in the two groups (127 versus 131 days), but the weight $\mathrm{z}$ score at discharge was lower in the SGA group $(-3.6$ versus -1.7$)(p=0.001)$. Indeed, there was no significant difference in the change in $\mathrm{z}$ score from birth between the two groups (median -1.53 versus $-1.07, \mathrm{p}=0.306)$.

Conclusions Amongst infants with a BW $<750$ gm, SGA compared to AGA suffered greater morbidity, but not mortality. These data are important for counselling parents as, in this population, any advantage of later gestation may be negated by being SGA.

\section{REFERENCES}

1. Peacok JL, et al. Pediatr Res 2013;73:457-63.

2. Tsai L-Y, et al. Pediatr Neonatol 2015;56:101-7.

\section{G203(P) UK NEONATAL RESUSCITATION SURVEY}

E Charles, K Hunt, A Milner, A Greenough. Women and Children's Health, School of Life Course Sciences, Faculty of Life Sciences and Medicine, King's College London, London, UK

\subsection{6/archdischild-2018-rcpch.198}

Aim In the UK, neonatal resuscitation practice follows national guidelines set by the UK Resuscitation Council. Two previous surveys, however, found significant differences in practice between units according to the level of care offered. ${ }^{1,2}$ Since then changes have been made to the guidelines ${ }^{3}$ and there has been interest in the role of carbon dioxide (CO2) monitoring during neonatal resuscitation. ${ }^{4}$ Our aim was to determine if these changes had altered resuscitation practice in the delivery suite across the UK.

Methods An online questionnaire was sent to the lead consultants of 189 units. If no response was received, a follow up email was sent and further non-response was followed by a telephone call. The results were compared to the 2012 survey. ${ }^{1}$

Results Over all, there was an $83 \%$ response rate per level of unit: (neonatal intensive care unit (NICU) 93\%, local neonatal unit (LNU) 83\%, special care baby unit (SCBU). Currently all units used an initial inspired oxygen ( $\mathrm{FiO} 2)$ of 0.21 for term born infants, whereas previously it had been used in $84.5 \%$ NICUs, $39.5 \%$ of LNUs and $31.7 \%$ of SCBUs. An initial FiO2 of 0.21 for preterm infants was used in NICUs, LNUs and SCBUs in 2017 in $86 \%, 93 \%$ and 93\% respectively and in 2012 in $42 \%, 22 \%$ and $24 \%$ respectively. Routine use of oxygen saturation monitoring for preterm infants had increased from $71 \%, 65 \%$ and $42 \%$ in 2012 to $94 \%, 88 \%$ and $84 \%$ (NICUs, LNUs and SCBUs respectively). CO2 monitoring was also more common, currently used by $88 \%, 81 \%$ and $84 \%$ compared to $24 \%-20 \%$ and $10 \%$ in 2012 (NICUs, LNUs and SCBUs respectively).

Conclusion There have been marked changes in delivery suite resuscitation practices since 2012 and more consistency across levels on units.

\section{REFERENCES}

1. Murthy V, et al. Arch Dis Child Fetal Neonatal Ed 2012;97:F154-5.

2. Mann C, et al. Resuscitation 2012;83:607-11.

3. Wyllie J, et al. European Resuscitation Council Guidelines for Resuscitation 2015.

4. Hawkes G, et al. Resuscitation 2014;85:1315-9. 